\title{
Posibilidades educativas de la wiki
}

\section{Educational possibilities of wiki}

Francisco Mora Vicarioli'

Fecha de recepción: 31 de mayo del 2011

Fecha de aprobación: 5 de febrero del 2012

Mora, F. Posibilidades educativas de la wiki. Tecnología en Marcha. Vol. 25, No 3. Julio-Setiembre 20 |2. Pág | |3-1 |8. 


\section{Resumen}

El artículo presenta el recurso wiki como una herramienta flexible que aporta grandes posibilidades a la educación. Su principal característica es que permite a un conjunto de usuarios interactuar de forma asincrónica para crear algún tipo de contenido y que cada uno puede agregar, quitar o editar los aportes propios o de los demás.

\section{Palabras clave}

Wikis, educación, web 2.0 y aprendizaje colaborativo.

\section{Abstract}

The article presents the use of wiki as a flexible tool that provides great opportunities for education. The main feature of this resource is that it allows a set of users to interact asynchronously to create some content and everyone can add, remove or edit the contributions from themselves or others.

\section{Key words}

Wikis, education, web 2.0, collaborative learning.

\section{Introducción}

El nacimiento del recurso wiki es parte de la gran cantidad de novedades que nos trajo la web 2.0. Dicho recurso posee características que lo hacen un medio eficaz para desarrollar trabajos colaborativos y propiciar un papel activo por parte del usuario, ya que es posible modificarlo las veces que sea necesario e interactuar con distintos participantes de forma asincrónica. Además, en la mayoría de los casos, la interfaz de las wikis es totalmente amigable y no requiere conocimientos avanzados de informática. Todo lo anterior hace de la wiki un recurso valioso para generar actividades en el contexto de la educación a distancia y en general en otros contextos educativos.

El uso del recurso wiki logra hacer que el docente sea coherente con los vertiginosos avances tecnológicos, si se toma en cuenta que los estudiantes de hoy son nativos digitales y se sienten más motivados al utilizar herramientas tecnológicas. La herramienta wiki, por su naturaleza virtual, constituye un valioso apoyo para el estudiante y un lugar donde se pueden desarrollar trabajos o proyectos de forma virtual.

En este artículo se describen aspectos generales del recurso wiki, se destacan sus características y se recomiendan algunos espacios gratuitos donde se pueden conformar wikis para distintos propósitos, entre ellos el educativo, en el cual se pueden generar dinámicas valiosas.

\section{La wiki como herramienta de la web 2.0}

Antes de la web 2.0, el papel del usuario de internet era el de simple lector, ya que no podía contribuir con contenidos o alterarlos; "los usos de la red estaban orientados en torno a una concepción estática y expositiva, con exiguas posibilidades de interacción" (Villarroel, 2007). Con la introducción de la web 2.0 surgen nuevas aplicaciones: internet ya no se limita solo a contener información sino que ofrece recursos que implican un papel activo de los usuarios, al permitirles generar trabajo colaborativo de forma asincrónica, pero manteniendo siempre un registro de todo aquello que se agrega o elimina.

Con esto, el contexto educativo se beneficia enormemente. Al respecto, García (2009) indica que "la llegada de internet y sus múltiples herramientas (correo electrónico, listas de distribución, chat, blogs, news, wikis) dan un nuevo significado y poder a la educación a distancia, generando la posibilidad de enseñar y aprender a través de la red".

En el contexto actual, "con la difusión de internet, la red ha ido ofreciendo herramientas que fomentan la interactividad, el aprendizaje cooperativo, la adquisición de conocimientos de manera dinámica, etc." (Gimeno, 2009). Entre estas herramientas se encuentra la wiki, la cual, por sus características técnicas, puede potenciar algunas actividades que los profesores pueden asignar a sus estudiantes de forma virtual.

Las tecnologías wikis "propician la descentralización y disparan la creatividad colectiva" (Rey, 2009). Lo anterior es posible porque, gracias a las características propias del medio, cada usuario puede agregar, editar y borrar información a su gusto, así como vincular múltiples recursos multimedia. 
En relación con su uso, la wiki es parte de otra serie de recursos de internet en los cuales "el conocimiento se ve más abierto gracias a aplicaciones, programas y páginas web que no requieren grandes conocimientos técnicos informáticos y facilitan la integración de contenidos de manera abierta y democrática" (Gimeno, 2009).

En este sentido, la novedad que introducen es que son herramientas fáciles de usar: no es necesario que el usuario tenga grandes habilidades informáticas; "son aplicaciones interactivas ya que favorecen al máximo la formación de redes comunicativas, presentándose, incluso, como herramientas dirigidas al trabajo cooperativo" (Villarroel, 2007). El aspecto mencionado brinda grandes posibilidades para aquellas personas que desean utilizar el medio electrónico para interactuar y generar grupos de discusión sobre temáticas variadas.

Es importante destacar que estas aplicaciones de la web generalmente son gratuitas y fáciles de crear, lo cual ofrece enormes posibilidades para instituciones y docentes que no pueden costear el pago de plataformas de aprendizaje en línea y que, por medio de recursos gratuitos, pueden generar excelentes dinámicas para propiciar el trabajo colaborativo.

\section{¿Qué es una wiki?}

En términos sencillos, la wiki es un sitio donde el usuario puede editar el contenido de forma rápida; de hecho, la palabra wiki proviene del hawaiano y significa "rápido". Se le llama wiki a las páginas web con enlaces, imágenes y cualquier tipo de contenido que pueden ser visitadas y editadas por cualquier persona. Por medio de una barra de edición, es posible agregar contenidos, videos y enlaces, crear vínculos, páginas nuevas y generar un menú de navegación, entre otras funciones.

Es importante destacar que la mayoría de las wikis tienen una forma de establecer plantillas y otras funcionalidades a lo largo de todo el sitio. También permiten gestionar permisos de usuario a nivel de sitio y de página, lo cual indica que esta simple herramienta puede generar un entorno para gestionar el aprendizaje en línea de forma sencilla y gratuita (González, Calderón, Galache y Torrico, 2007).
Un aspecto que tienen las wikis, y que es una de sus características principales, es el del seguimiento, en especial si se trata de wikis para la elaboración de trabajos de forma colaborativa. Al respecto, la mayoría de servicios de wiki permiten registrar un historial de ingreso, en algunos casos con un "control de cambios", es decir, se registra todo aquello que fue cambiado por cada uno de los usuarios $y$, cuando el usuario lo solicite, se envía un informe automático a los usuarios registrados con el fin de mantenerlos al tanto.

Este es un aspecto de interés para el educador que desea verificar el avance en alguna asignación de forma totalmente en línea. Las wikis, al registrar la "historia" de construcción de un documento, ofrecen a los docentes una información altamente valiosa para realizar el seguimiento del proceso de aprendizaje tanto individual como grupal de los estudiantes" (Area, 2009).

\section{Origen del recurso wiki}

La primera wiki se originó en la década de 1990. El primer sistema wiki nació gracias a Ward Cunningham en 1995 (Cabero y Román, 2006). Por lo tanto, es un recurso que tiene más de una década de existir, lo cual le ha permitido evolucionar junto con la tecnología, convirtiéndose en una herramienta que ha mejorado notablemente en términos de flexibilidad, además del gran crecimiento de sus usuarios.

Se debe anotar que el uso de algunas wikis se ha generalizado de forma masiva, como es el caso de Wikipedia, "la famosa enciclopedia libre y colaborativa" (Cabero y Román, 2006). Este tipo de ejemplo refleja el impacto que puede tener un recurso al cual las personas pueden aportar su conocimiento sobre cualquier área del saber de forma libre, y refleja por qué es posible encontrar todo tipo de información en esta enciclopedia.

Desde el origen del recurso, cada vez son más los servicios de wikis en el mercado; algunos son gratis, otros de pago. En el sitio Wiki Matrix (http://www. wikimatrix.org/), es posible visualizar cerca de 133 servicios de wikis en internet y comparar de forma gratuita sus características, además, los usuarios pueden constatar la gran proliferación de dicho recurso. 


\section{Ventajas de las wikis}

Las wikis pueden crearse y colocarse a disposición de un grupo cerrado de usuarios o hacerse públicas. Estas permiten llevar un control de los cambios realizados, que son registrados en la propia herramienta de forma automática. Más aún, algunos servicios de wikis tienen la opción de que cada cambio sea notificado vía correo electrónico a los usuarios. Además, el creador de la wiki tiene la potestad de eliminar la posibilidad de edición a uno o varios miembros del grupo.

Como indican Ruiz, Sánchez y Palomo (2008), entre las ventajas de las wikis están:

- La página se escribe y modifica de forma rápida.

- La interfaz es fácil de navegar y se maneja de manera intuitiva.

- Permite, por el carácter abierto de sus contenidos, un uso colectivo.

- No se necesita conocimiento de ningún tipo, ya que presenta un editor en línea para su uso.

Otra ventaja importante es que no se requiere instalar ningún software extra, pues la página se abre con cualquier navegador de internet. Además, se debe anotar que el hecho de que sean recursos gratuitos permite que gran cantidad de personas pueda acceder a ellos, sin necesidad de invertir en servidores o en software para tener sitios propios.

\section{Desventajas de la wiki}

Las mayores desventajas que tiene la wiki, y que se derivan de su libertad de edición, es la pérdida de información a causa de usuarios que deciden borrar el contenido y la incorporación de materiales con derechos de autor, lo cual provoca problemas legales. Estos inconvenientes se pueden solucionar, ya que, como se indicó, la wiki registra los cambios, por lo que eventualmente se podría determinar cuáles usuarios generaron daños; también se puede acceder a versiones anteriores y eso facilitaría la reparación de los mismos.

Otra de las desventajas es que en la mayoría de los entornos gratuitos de las wikis se tiene que lidiar con la publicidad generada por los sitios para sostener el servicio, lo que puede distraer al usuario.
La wiki requiere de usuarios comprometidos, que deseen colaborar para que las asignaciones que se realicen en este tipo de recurso sean exitosas. Para generar espacios que logren el aprendizaje colaborativo, se requiere de estrategias por parte de los facilitadores que realmente promuevan la contribución y la adecuada interacción.

A continuación, se brindan algunas recomendaciones para el uso de las wikis en el contexto educativo.

\section{Aplicaciones en la educación}

Las aplicaciones de las wikis en la educación son muchas y dependen de la estrategia que se siga y del tipo de mediación que se utilice, de manera que las actividades que se generen sean atractivas para el estudiantado.

Existen propuestas de uso de la tecnología wiki en distintos tipos de actividades educativas (Giménez y González, 2006; Melero y Tricas, 2005; González et al., 2006): "como herramienta de trabajo usada por el docente para elaborar material para el alumnado o como herramienta para que los estudiantes, guiados y corregidos por el profesor, elaboren apuntes o ejercicios de una asignatura" (Villanueva, 2009).

Por otro lado,"la opción de compartir conocimientos a través de las wikis es otra de las grandes posibilidades que estamos encontrando en este momento en el campo de la educación" (Cabero y Román, 2006), debido a la flexibilidad de que se dispone para compartir recursos, contenidos, vínculos y diferentes aspectos que sirven para un determinado curso o asignatura.

Como se mencionó anteriormente, la facilidad de edición es un aspecto básico de la wiki, pues "refleja un grado de 'democratización' en el aprendizaje, ya que no se basa en una (excesiva) preocupación por la autoría y facilita la interacción entre pares conformando un producto en constante cambio que fluye inacabado" (Barberá, 2009).

Ya que la wiki se perfila como un recurso apropiado para realizar trabajos en equipo, se debe tener presente que:

Desde la perspectiva del aprendizaje de los estudiantes, las wikis son recursos útiles para el desarrollo de proyectos de investigación en una perspectiva socioconstructivista del conocimiento. 
Esta herramienta permite, por una parte, que un equipo de alumnos redacten, escriban y reconstruyan la información y conocimiento que van elaborando en torno a un determinado tópico, problema o caso planteado de forma colectiva. (Area, 2009).

Las wikis logran una dinámica que difícilmente se consigue en el entorno presencial, en el sentido del registro de los aportes de cada participante, dado que en ellas los usuarios pueden interactuar de forma asincrónica y dejar registrados todos sus aportes. El profesorado puede sacar provecho de estas características para ver el avance de los estudiantes en una determinada tarea o proyecto que involucre el uso de una wiki. Se debe tener presente que dentro del entorno virtual de la wiki, los estudiantes deben ser monitoreados de tal forma que exista respeto por los criterios de los demás.

Con respecto al papel de los estudiantes, lo que se busca es que se involucre activamente en el proceso de enseñanza-aprendizaje. En este sentido, las wikis propician que el estudiante del modelo a distancia tenga un papel protagónico en su proceso, sin necesidad de contar con herramientas sofisticadas como son los entornos virtuales de aprendizaje (EVA).

También se debe mencionar que, en estos momentos, algunos entornos virtuales de aprendizaje, como es el caso de Moodle (http://moodle.org/?lang=es_es), poseen su propia wiki internamente, lo cual permite que en un curso virtual el facilitador elija dinámicas que involucren el uso de wikis.

Con el uso de la wiki, cada estudiante aporta según sus posibilidades y a su propio ritmo; incluso, es posible que en estos entornos las personas menos expresivas logren integrarse y participar y comunicarse con otros estudiantes.

Como se anotó, la wiki no requiere conocimientos complejos de informática por parte del usuario y tiene la ventaja de necesitar poca explicación sobre su uso, ya que los aspectos básicos que se pueden hacer en ella aparecen a la vista del usuario. Esto favorece al profesor, ya que no debe dedicar demasiado tiempo a los aspectos operativos 0 aclarando dudas técnicas.

Es importante que los docentes que deseen implementar la herramienta de la wiki investiguen las características de los distintos proveedores, para seleccionar la que se ajuste mejor a sus necesidades. Una vez hecha la selección, es deseable que el docente haga una presentación completa del recurso para que los usuarios sepan las posibilidades y las limitaciones que ofrece el mismo.

Por último, las diversas funciones de seguimiento de la wiki permiten que el profesor facilitador pueda evaluar el proceso de forma sencilla, ya que se evidencian los aportes de cada uno de los usuarios: "la wiki tiene una importante potencialidad evaluativa, ya que ofrece información detallada sobre el 'proceso' de desarrollo de cualquier tarea o producto solicitado a los estudiantes, así como información sobre las aportaciones individuales de cada alumno dentro de su grupo de trabajo" (Area, 2009).

De forma general, las características anteriores reflejan cómo el medio puede ser muy versátil y dotar al docente de actividades que motiven y logren que los estudiantes tengan un papel activo en el proceso.

\section{Algunos espacios gratuitos para la creación de wikis}

En la tabla I se presentan algunos espacios gratuitos disponibles en internet para la creación de wikis. Todos son de carácter gratuito, pero también ofrecen opciones de pago para que el usuario pueda acceder a otras funciones.

Como se mencionó anteriormente, existe gran cantidad de servicios de wiki en internet (ver sitio de wiki matrix http://www.wikimatrix.org/), por ello

Tabla I. Espacios gratuitos de wikis disponibles en internet

\begin{tabular}{|l|l|}
\hline Wikispaces & http://www.wikispaces.com/ \\
\hline @Wiki & http://atwiki.com/ \\
\hline Clearwiki & http://www.clearwiki.com/ \\
\hline Zoho Wiki & http://wiki.zoho.com/login.do?serviceurl=\%2Fregister.do \\
\hline Wiki dot & http://www.wikidot.com/ \\
\hline
\end{tabular}


el usuario debe seleccionar aquel que se ajuste a sus necesidades. Un aspecto importante de tomar en cuenta es la facilidad de creación del sitio y la facilidad que ofrece el entorno para elaborar dinámicas colaborativas.

\section{Conclusiones}

- El recurso wiki proporciona una gran gama de posibilidades a docentes y a estudiantes, todas enfocadas en un aprendizaje colaborativo. Esto es fundamental si se quiere propiciar un estilo de aprendizaje activo.

- Es posible encontrar múltiples proveedores de wikis de forma gratuita en internet.

- Debido a la naturaleza editable de la wiki, podría perderse el contenido por error de un usuario o vandalismo. En este caso, se debe tener presente que en algunas wikis se pueden recuperar los cambios realizados.

- La wiki tiene ciertas similitudes con una página web, pero a diferencia de esta, no requiere conocimientos de programación y posee un entorno fácilmente modificable.

\section{Bibliografía}

Area, M. (2009). Las wikis en mi experiencia docente. Del diccionario de la asignatura al diario de clase. Recuperado el 18 de agosto del 2010, desde http:// revistas.um.es/red_u/article/view/9274I.

Barberá, E. (2009). Filosofía Wiki: el compromiso de las soluciones. RED-Revista de Educación a Distancia. Recuperado el 18 de agosto del 2010, desde http:// www.um.es/ead/red/MI l/intro.pdf.

Cabero, J. \& Román, P. (2006). E-actividades: un referente básico para la formación en internet. Sevilla: Editorial MAD.
Falla, S. (2007). ¿Qué es un wiki? Recuperado el 18 de agosto del 2010, desde http://www.sld.cu/galerias/pdf/ sitios/bmn/que_es_un_wiki.pdf.

García, L. (2009). Concepción y tendencias de la educación a distancia en América Latina. Recuperado el 15 de agosto del 20l0, desde http://www.oei.es/ DOCUMENTO2caeu.pdf.

Gimeno, A. \& García, J. (2009). Wikis y el nuevo estudiante de lenguas extranjeras. Recuperado el 18 de agosto del 2010, desde http://www.um.es/ead/red/Ml2/7GimenoGarcia.pdf.

González, A., Calderón, S., Galache, T. \& Torrico, A. (2007). Uso de wikis para la realización de trabajos colaborativos en el aula. Recuperado el 18 de agosto del 2010, desde ht

Lupion, P. \& Rama, C. (20।0). La Educación Superior a Distancia en América Latina y el Caribe: Realidades y tendencias. Recuperado el 18 de agosto del 2010, desde http://sitio.flacso.edu.uy/wp-content/ uploads/2009/I 2/Libro_EduDist2009.pdf.

Macías, C. (2007). Les wikis: espaces del l'intelligence collective. de Jérome Delacroix. Recuperado el 13 de agosto del 2010, desde http://redalyc.uaemex.mx/ redalyc/pdf/547/5470 1704.pdf.

Rey, A. (2009). Innovación 2.0: web 2.0 y gestión de la innovación. Recuperado el 21 de marzo, 2009, de http://www.emotools.com/static/upload/files/ Innovacion20_I.pdf.

Ruiz, J., Sánchez, J. \& Palomo, R. (2008). Posibilidades educativas de la Web 2.0. Recuperado el 22 de marzo del 2009, desde http://tecnologiaedu.uma.es/ materiales/web20/archivos/cap9Web20_wikis.pdf.

Villanueva, A. (2009). Uso de wikis en Ingeniería Informática. Recuperado el 18 de agosto del 20l0, desde http:// www.um.es/ead/red/MI / / 0-Villanueva.pdf.

Villarroel, J. (2007). Usos didácticos del wiki en educación secundaria. Recuperado el 22 de marzo del 2009, desde http://www.ehu.es/ikastorratza/I_alea/wiki.pdf. 